\title{
Betreuung und Pflege bei Demenz - die Rolle der Angehörigen
}

\author{
Rosemarie Drenhaus-Wagner
}

(C) Springer-Verlag 2012

Eine Demenz betrifft nicht nur den Erkrankten selbst, sondern auch seine Angehörigen - ganz besonders seine Hauptpflegeperson, die im Verlaufe der Krankheit in unterschiedlicher Weise gefordert wird: Zu Beginn der Demenz wissen weder der Betroffene noch seine Angehörigen, dass die ersten Anzeichen - und daraus resultierende verletzende Verhaltensweisen - auf eine ernsthafte Erkrankung zurückzuführen sind. Versäumnisse und Verweigerungen des Erkrankten verstehen Angehörige dann als irritierende Unaufmerksamkeiten oder gar Böswilligkeiten. Misstrauen und Beschuldigungen werden persönlich genommen. Vermeidungsverhalten des Erkrankten und seine Neigung, Fehlleistungen zu vertuschen bzw. anderen anzulasten, wird missverstanden, was zu weiteren Spannungen führt. Diese lassen Angehörige verzweifeln und rufen beim Ehepartner gelegentlich sogar den Wunsch nach Trennung hervor. Damit findet sich der Angehörige zunächst in einer Opferrolle wieder.

Der fortschreitende Krankheitsverlauf führt beide aus der gemeinsamen Vertrautheit in die gegenseitige Fremdheit. Bis zur völligen Selbstaufgabe gleichen Angehörige quasi als „Hilfs-Ich“ die zunehmenden Defizite des Erkrankten aus, um ihm Halt und Sicherheit zu geben. Dabei übernimmt die Hauptpflegeperson nicht nur frühere Rollen des Erkrankten, sondern auch noch eine völlig neue Rolle, nämlich die eines beschützenden und fürsorglichen Elternteils.

Im späten Stadium sind Angehörige vor allem als Pflegeperson gefordert. Hierzu fehlt ihnen meist jede Ausbildung. Die Folge sind körperliche und seelische Überlastungen, die psychosomatische Beschwerden verursachen.

All diese Belastungen $\mathrm{zu}$ bewältigen, erfordert viel Kraft. Deshalb müssen Angehörige diese effizient einset-

R. Drenhaus-Wagner $(\bowtie)$

Alzheimer Angehörigen-Initiative e. V., Berlin, Deutschland zen, indem sie z. B. früh lernen, mit der Krankheit zu leben, statt erfolglos gegen sie anzukämpfen. Angehörige sind gut beraten, frühzeitig Rat zu suchen und Hilfe anzunehmen. Dauerhafte Begleitung und zeitweise Entlastung durch eine Alzheimer-Organisation vermitteln Sicherheit und schaffen wieder ein Stück persönlichen Freiraum. Hilfsangebote wie Tanznachmittage und betreute Urlaube gehen über die Entlastung hinaus. Die so ermöglichte soziale Teilhabe erlaubt wieder ein Stück Normalität zu erleben, nicht selten sogar Momente der Freude - trotz der Demenz. 